\title{
RAINER MARIA RILKE UND VILHELM HAMMERSH $\varnothing I$. ZUR BEZIEHUNG ZWISCHEN BILDENDER KUNST UND DICHTUNG ZUR ZEIT DER JAHRHUNDERTWENDE
}

\author{
Steffen Arndal ${ }^{*}$ \\ Syddansk Universitet, Dänemark
}

\begin{abstract}
Resumo: Der Aufsatz untersucht und wertet den Eindruck, den zwei Gemälde des dänischen Malers Vilhelm Hammershøi (1864-1916), das "Selbstporträt" (1889) und das Porträt seiner Verlobten Ida Ilsted, "Porträt eines jungen Mädchens" (1892), auf Rilke gemacht haben. Das geschieht im Rahmen seiner Bewunderung für den dänischen Dichter Jens Peter Jacobsen (1847-1885). Dabei dient das Schauen als gemeinsamer Referenzpunkt von bildender Kunst und Literatur. Rilke war bei seinen wiederholten Besuchen in Kopenhagen während seines Aufenthaltes in Schweden 1904 hauptsächlich durch seine Bewunderung für Jens Peter Jacobsen geleitet. Der Eindruck der Kunst Vilhelm Hammershøis, die er auf der Durchreise nach Schweden durch dessen Porträt seiner Verlobten Ida Ilsted in Düsseldorf empfing, machte es jedoch möglich, dass Hammershøi quasi als "stand in" für den verstorbenen dänischen Dichter wirken konnte. Rilke erlangte Zugang zu der Kunstsammlung von Hammershøis Mäzen Alfred Bramsen und damit die Möglichkeit, weitere Gemälde von Hammershøi zu studieren. Die Analyse von zwei Porträts macht es wahrscheinlich, dass es vornehmlich das bewusstere Schauen der beiden Personen war, das Rilke interessierte. Im "Selbstporträt" ein konzentriertes, distanziert sachliches, gleichsam horchendes Schauen, im Porträt Ida Ilsteds ein unschuldiger, inhaltsloser, offener Blick auf der Kippe zwischen Innen und Außen. Die beiden Porträts thematisieren damit das bewusstere Schauen, das Rilke bei Jacobsen bewunderte, und weisen gleichzeitig auf die Bedeutung des Schauens in künftigen Werken Rilkes und in den Briefen über Cézanne.
\end{abstract}

Palavras-chave: Rilke. Hammershøi. Jacobsen. Poesie. Bildender Kunst.

Der dänische Maler Vilhelm Hammershøi (1864-1916), der in seiner Gegenwart fast nur von einem kleineren Kreis von Kunstkennern in Kopenhagen geschätzt wurde, ist in den letzten Jahrzehnten der Gegenstand eines wachsenden Interesses geworden, das durch mehrere internationale Ausstellungen und eine wachsende Zahl von Publikationen zum Ausdruck gekommen ist. In der Tradition der dänischen Interieurmalerei des frühen 18.

EY NC No Esta obra está licenciada sob uma Licença Creative Commons.

*Steffan Arndall arbeitet als Hochschullehrer an der Syddansk Universitet in Dänemark.

Anu. Lit., Florianópolis, v. 19, n. 1, p. 224-232, 2014. ISSNe 2175-7917 
Jahrhunderts, des sogenannten "goldenen Zeitalters" der dänischen Malerkunst, verwurzelt gilt Hammershøi schon seit dem Ende des 19. Jahrhunderts als "der Maler der stillen Stuben". Dabei verleihen aber seine Tendenz zur Abstraktion, seine leeren Räume mit ihren vereinzelten Gegenständen und seine seltsam anonymen Porträts seinen Gemälden ein eigentümlich modernes Gepräge, das den herrschenden Geschmack seiner Gegenwart provozierte. Es ist für die Modernität Hammershøis kennzeichnend, daß es die Auseinandersetzungen über zwei Gemälde ${ }^{1}$ von ihm war, die 1891 zur Bildung der dänischen Sezession, "Den Frie Udstilling", führte.

\section{Selvportrat [1889] e Portrat af en ung pige (Ida Ilsted) [1890]}

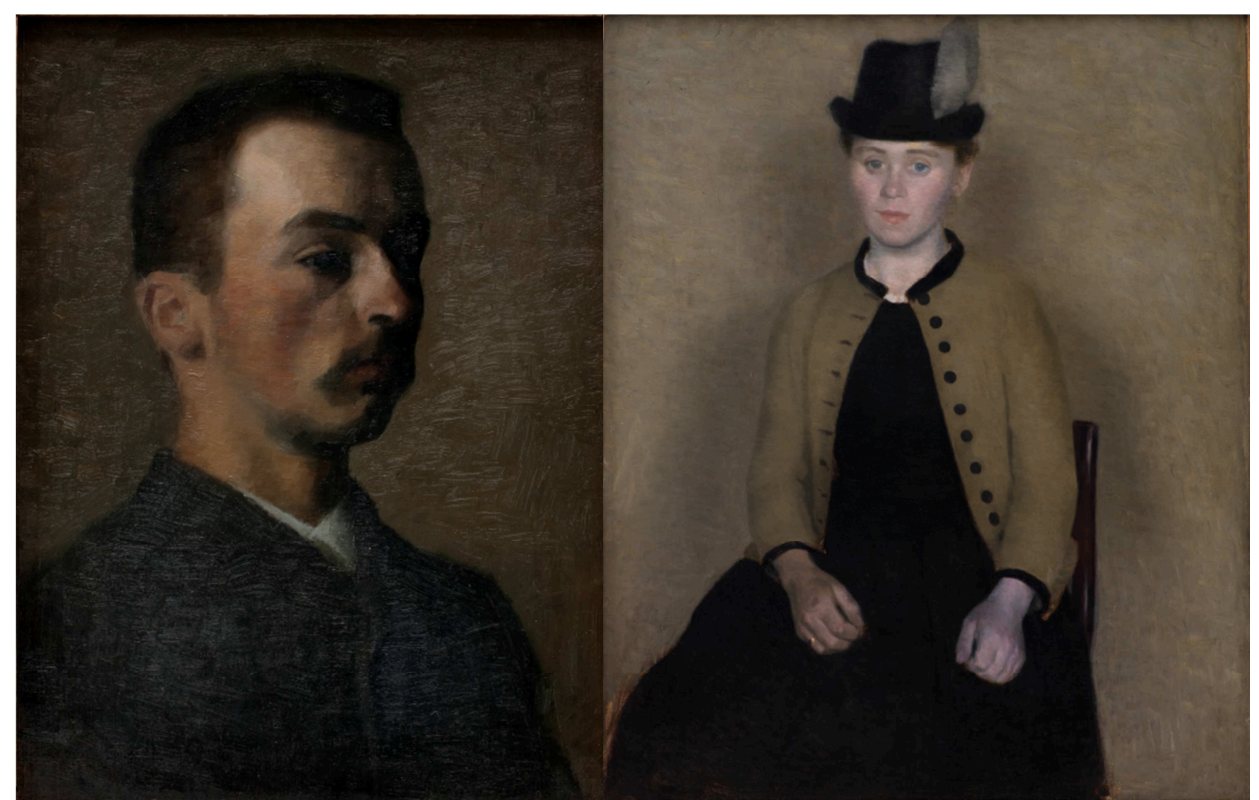

Fonte: Statens Museum for Kunst, National Gallery of Denmark, Danmark / Denmark, www.smk.dk

E Diese Spannung zwischen Tradition und Modernität erschwert in hohem Maße eine nähere Bestimmung von Hammershøis Eigenart und eine überzeugende Einordnung seiner Kunst in die kultur- und geistesgeschichtlichen Strömungen der Jahrhundertwende. Über die technische Seite, seine Motivwahl, seine eigentümliche grautönige Farbenskala und seine abstrahierende Betonung der Komposition und der linearen Spannungen sowohl auf der Bildfläche als auch in der Tiefendimension herrscht weitgehend Einigkeit. Wenn es aber gilt, Hammershøis Auffassung des Menschen und der Wirklichkeit näher zu bestimmen, gehen die Meinungen auseinander. Einerseits wird die introvertierte Einsamkeit und Isoliertheit von

\footnotetext{
${ }^{1}$ Das "Portræt af en ung Pige" (Anna Hammershøi) dem 1885 die Neuhausensche Prämie verweigert wurde, was ein Protestbrief junger Künstler an die Akademie hervorrief, und "Ung Pige, der syer", das 1888 von der Charlottenborgausstellung refusiert wurde.
} 
Hammershøis Menschen betont, und seine stillen Stuben werden entsprechend etwa als klaustrophobisch bezeichnet oder durch ihre eiskalte, gespenstische Leere charakterisiert. Andererseits wird Hammershøis Gemälden ein mystischer Charakter verliehen. Man spürt in ihnen eine rätselhafte Anwesenheit, die nicht zuletzt im Zusammenhang mit den Interieurs als magischer Realismus bezeichnet wird. Diese widersprüchlichen Deutungen verbinden sich oft mit einer Tendenz, Hammershøis Gemälde zu psychologisieren und in ihnen die Entfremdung und die Einsamkeit des modernen Menschen oder die soziale Situation der Frau thematisiert zu sehen.

Da Hammershøi sich weder über seine eigenen Werke noch über psychologische und weltanschauliche Probleme geäußert hat und seinen Nachlaß, der etwas über diesbezügliche Fragen enthalten haben könnte, vernichten ließ, bietet sich als Möglichkeit, der Gefahr einer verallgemeinernden, stereotypen Psychologisierung zu begegnen, nur eine wechselseitige Erhellung der Künste an. Durch eine vorsichtig deutende Beziehung der Kunst Hammershøis auf gleichzeitige literarische Strömungen lassen sich nicht nur ein präziseres Verständnis ihrer Eigenart erzielen, sondern auch aufschlussreiche Deutungsaspekte der gleichzeitigen Dichtung gewinnen.

Die Wechselwirkung zwischen bildender Kunst und Dichtung war gerade zur Zeit der Jahrhundertwende von besonderer Bedeutung. Ein herausragendes Beispiel dafür ist bekanntlich Rainer Maria Rilke. Seine impressionistisch zufällige, von stimmungshafter Emotionalität und einer Spannung von dekadenter Müdigkeit und lebensbejahendem Vitalismus bestimmte Jugendlyrik steht weitgehend im Banne des Jugendstils, der sich auch in dem Aufsatz Worpswede (1903, entst. 1902) geltend macht. Sein Aufsatz über Auguste Rodin (1903, entst. 1902) leitete die Wende zu einem bewussteren Schauen und einer objektiveren und konkreteren Dichtung ein, wobei mit Karl E. Webb betont werden muss, daß Rilkes Beschreibungen Rodinscher Skulpturen vielfach noch mit den Kategorien des Jugendstils arbeiten. Der Einfluss Rodins ist vor allem in den Dinggedichten der Neuen Gedichte (1907) zu beobachten, während Der neuen Gedichte anderer Teil (1908) auch von dem Erlebnis Cézannes auf der großen retrospektiven Ausstellung in Paris 1906 mitbestimmt ist. Auch in den seit 1904 entstehenden Aufzeichnungen des Malte Laurids Brigge (1910) ist der Einfluß der bildenden Kunst unter anderem im Zusammenhang mit dem Begriff des Schauens entscheidend, wobei nicht zuletzt die Raumperzeption Cézannes von Bedeutung zu sein scheint. Als Rilke 1904 von Rom aus die Reise nach Skandinavien antrat, die ihn mit Hammershøi bekannt machen sollte, befand er sich - nach dem Rodinaufsatz, während die 
Neuen Gedichte im Entstehen und der Malteroman angefangen waren, aber vor der Begegnung mit der Kunst Cézannes - in einer Phase seiner Entwicklung, wo er sich erst allmählich aus den Ranken des Jugendstils und der dekadenten Stimmungsdichtung loslöste und, durch das Rodinsche toujours travallier geleitet, sich bemühte, ein ernsteres, auch das Schauen umfassende "Arbeiten" zu erlernen.

Dasselbe erhoffte er sich durch die geplante intensive Beschäftigung mit Jens Peter Jacobsen. Rilke unternahm von Schweden aus mehrere kurze Reisen nach Kopenhagen und erlebte die Stadt und ihre Einwohner durch die Brille seiner Jacobsenbegeisterung. Wie er seiner Frau Clara schreibt, sehe er Kopenhagen "durch I.P. Jacobsen und um seinetwillen. Es ist nicht weit, aber es ist leise,- es ist altmodisch und so ganz und gar Interieur, der Stimmung nach" ${ }^{2}$.

Jacobsens Vorbild folgend wollte er hier gerade "arbeiten", nämlich Archivstudien in der Königlichen Bibliothek durchführen, und er bemühte sich, im Hinblick auf das geplante Jacobsenbuch die dänische Sprache zu erlernen. Dabei war Rilkes Bild des dänischen Dichters vom Anfang an in einem steten Wandlungsprozess begriffen, der von dem dekadenten "Mondpoeten" und nuancenreichen, dem Jugendstil verwandten Impressionisten in Richtung größerer Sachlichkeit führte. Vor allem scheint Jacobsens Fähigkeit, "sinnliche Äquivalente" [...] "auch für das Leiseste und Unfaßbarste in uns" zu finden, wie es ein paar Jahre später (1907) in einem Brief an den Buchhändler Hugo Heller heißt, entscheidend gewesen zu sein. ${ }^{3}$ Während des Aufenthaltes in Schweden vereitelten sich Rilkes Jacobsenpläne aber allmählich. Die dänische Sprache erwies sich als zu schwierig, und die Aufenthalte in Kopenhagen wurden u.a. von seinen Bemühungen, seiner Frau Clara einen Unterhalt zu verschaffen, in Anspruch genommen. Am Ende seines Skandinavienaufenthaltes plante Rilke auf der Durchreise im Dezember 1904 einen längeren Aufenthalt in Kopenhagen, jetzt aber nicht nur damit er sich "mit der Menge des Jacobsenmaterials bekannt" machen konnte, sondern, wie es fast gleichlautend in Briefen an Clara (18. Nov.) und an Lou Andreas Salomé (4. Dez.) heißt, "vor allem aber, um Hammershøi zu besuchen", bzw. "zu sehen".

Dass Hammershøi in dieser Weise quasi als stand-in für Jacobsen erscheint, hängt damit zusammen, daß Rilke auf der Reise nach Skandinavien in einer Kunstausstellung in Düsseldorf einen starken Eindruck von seiner Kunst empfangen hatte. Dabei handelte es sich aber nicht um Hammershøis Interieurs, sondern um ein Porträt, nämlich Hammershøis 1890

\footnotetext{
${ }^{2}$ Siehe zu den Briefzitaten R.M. Rilke: Gesammelte Briefe in sechs Bänden. Hrsg. v. Ruth Sieber Rilke und Carl Sieber, Leipzig 1936-1939.

${ }^{3}$ Rainer Maria Rilke, Sämtliche Werke, hrsg. v. Ernst Zinn, Sechster Band, Frankfurt am Main: Insel Verlag 1964, S. $1020-1022$.
}

Anu. Lit., Florianópolis, v. 19, n. 1, p. 224-232, 2014. ISSNe 2175-7917 
entstandenes Porträt seiner Verlobten, Ida Ilsted. Dieses Bild machte einen solchen Eindruck auf Rilke, daß er sich bei einem Besuch in Kopenhagen im September durch den dänischen Jugendstildichter Sophus Michaëlis bei Hammershøis Mäzen, dem Kunstsammler Alfred Bramsen einführen ließ. ${ }^{4}$ Bramsen besaß das Porträt und zahlreiche wesentliche Arbeiten Hammershøis. Darunter waren die Interieurs aber nur mit einer sehr begrenzten Zahl vertreten, da Bramsen sie eher als eine Art Broterwerb Hammershøis wertete. Es waren also nicht die stillen Stuben, leeren Räume und isolierten Dinge, sondern andere Aspekte von Hammershøis Kunst, die von Anfang an für Rilke im Vordergrund standen.

Bramsen gab Rilke und Clara, die zu Besuch war, die Gelegenheit, die Bilder Hammershøis "zu genießen", worauf Rilke sich bezieht, als er in einem Brief an Bramsen vom 22. November darum bittet, während des Aufenthaltes auf der Rückreise nach Deutschland die Gelegenheit zu bekommen, die Gemälde "wiederzusehen und aufmerksam zu studieren". Gleichzeitig fragt er Bramsen, ob Hammershøi, der bei dem ersten Besuch in London war, zurückgekehrt sei, und äußert den Wunsch einer persönlichen Begegnung mit ihm. Wie wichtig Hammershøi Rilke geworden war, geht aus den abschließenden Zeilen des Briefes hervor:

\begin{abstract}
Aus alle diesem soll eine Studie über den Künstler, später vielleicht ein selbständiger Essay erwachsen; auch fühle ich, wie große Förderung meinen eigensten dichterischen Arbeiten aus der direkten Berührung mit diesem Meister für mich entstehen könnte, dessen großes und bedeutendes Werk ich in meiner Bewunderung wesentlich $\mathrm{zu}$ erfassen glaube. (RILKE, R. M. Brief an Alfred Bramsen vom 22. November 1904, in: Povl Vad 1988, S. 404. In dänischer Übersetzung.)
\end{abstract}

Wie im Falle Rodins und Jacobsens und später Cézannes steht für Rilke Hammershøis Künstlerpersönlichkeit im Zentrum. Durch die persönliche Begegnung erhofft er sich eine intuitive Einsicht - in einem späteren Brief an Bramsen (10. Nov. 1905) spricht er geradezu davon, "eingeweiht, durchdrungen" zu werden - in Hammershøis Existenz als schaffenden, arbeitenden Künstler, wobei zum "wesentlichen" Erfassen seines Werkes zweifellos auch Jacobsens Fähigkeit des "unwählerischen Schauens" und des Schaffens von "sinnlichen Äquivalenten [...] auch für das Leiseste und Unfaßbarste in uns" mitklingt.

Es ist für Rilkes Versuch einer intuitiven Einstimmung in Hammershøis Wesen symptomatisch, dass eine intellektuelle Auseinandersetzung für ihn anscheinend nicht die

\footnotetext{
${ }^{4}$ So Povl Vad, Vilhelm Hammershфi. Vark og Liv, Kopenhagen 1988, S. 432 (Anm. 272), der Bramsens Aufsatz "Vilhelm Hammershøi. Meister der Farbe, in Europäische Kunst der Gegenwart III, Leipzig 1905, S. 85, zitiert. Vgl. auch Bramsens Aufsatz Weltkunst. Der dänische Maler Vilhelm Hammersh $\phi$, in Zeitchrift für bildende Kunst, Neue Folge, sechzehnter Jahrgang, Leipzig 1905, S. 176 - 189, S.178, wo neben Rilke Alfred Lichtwark unter den Bewundern Hammershøis gezählt wird.
} 
entscheidende Rolle spielte. Schon vor der persönlichen Begegnung heißt es in dem Brief an Clara (18. Nov.), dass er Hammershøi "sprechen und schweigen hören" möchte. Dieser Wunsch hat sich, was das Schweigen anbelangt, für Rilke auch erfüllt, da der von Natur sehr zurückhaltende und schweigsame Hammershøi nicht der deutschen Sprache mächtig war. Das Schweigen ist aber nicht nur ein Verstummen der lauten Worte. In ihm wird das Schweigen selbst, eben "das Leiseste und Unfaßbarste in uns", wie es über Jacobsen heißt, hörbar. Rilke fügt deshalb hinzu: "Man fühlt, daß er nur malt und nichts anderes kann oder will." Als Maler, d.h. durch sein Schauen, vernimmt Hammershøi im Menschen die unhörbare Sprache des stummen Lebens und macht sie durch die visuelle Sprache seiner Bilder fühlbar. Bei diesem Schauen handelt es sich aber nicht nur um eine intuitive Einstimmung in das stumme Leben, sondern um ein "eindringliches, hingebungsvolles", allen Eindrücken offenes, aber gleichwohl "unwählerisches", d.h. distanziertes, sachliches ${ }^{5}$ Schauen, wie es im Brief an Ellen Key vom 2. April 1904 über Rodin und Jacobsen, bzw. in dem späteren Brief an Hugo Heller über Jacobsen heißt.

Dieses Schauen hat Rilke in dem Selbstporträt Hammershøis, das sich in der Sammlung Bramsens befand, studieren können. Man hat den ernsten, fast überheblichen Charakter des Porträts als Ausdruck von "aristokratischem Radikalismus (WIVEL, 1996, S. 4) gedeutet, was aber abwegig ist. Vielmehr ist das Statuarische der Figur auf das konzentrierte, distanziert sachliche, auf die leisesten Nuancen gleichsam horchende Schauen zurückzuführen, wobei es eine Rolle gespielt haben dürfte, daß Hammershøi, um sich selbst im Halbprofil darstellen zu können, durch ein System von zwei die Seitenverkehrtheit wieder umkehrenden Spiegeln geschaut hat. Er sieht sich von außen, so wie andere Menschen ihn sehen. Entscheidend ist, daß Hammershøi auf jede Selbstanalyse verzichtet. Er malt sich selbst, ausschließlich wie er sich sieht, wodurch gerade das Anonyme, rätselhaft Fremde und Beunruhigende der menschlichen Persönlichkeit in dem Bild zum Vorschein kommt, und zwar in der Form einer "wesentlichen Schlichtheit", die Rilke nach der persönlichen Begegnung in einem Brief an Clara (4. Dez.) an Hammershøi rühmt. Rilke bezieht sich dabei auf die Selbstporträts, die er und Clara bei dem ersten Besuch bei Bramsen gesehen hatten, weil Hammershøi sich in der Zwischenzeit einen Vollbart zugelegt hatte:

Gestern habe ich zum erstenmal Hammershøi gesehen ... Er ist anders als auf seinen Selbstporträts, älter und weniger schlicht, weil langes Haar und ein vollwachsender Bart das Gesicht über seine einfachen Konturen hinaus erweitern und scheinbar komplizieren. Aber ich bin sicher, je öfter man ihn sieht, desto deutlicher wird man

\footnotetext{
${ }^{5}$ Vgl. den Begriff des "sachlichen Schauens" im Malteroman und seine Bedeutung für das "sachliche Sagen".
} 
ihn erkennen, und desto mehr wird man seine wesentliche Schlichtheit finden. (Rilke Brief an Clara Westhof vom 4. Dez. 1904, vgl. Anm. 2).

Die "wesentliche Schlichtheit" bezieht sich sowohl auf Hammershøis Persönlichkeit als auch auf seine Kunst. Dabei befindet sich die Betonung der Schlichtheit und der "einfachen Konturen" in deutlicher Übereinstimmung mit Rilkes Bewertung der Kunst Rodins und Jacobsens, etwa in dem Brief vom 2. April an Ellen Key, wo es heißt: "Beide haben Dinge gemacht. Dinge mit lauter sicheren Grenzen." Wenn Rilke dann hinzufügt: "und unzähligen Überschneidungen und Profilen", so befindet er sich anscheinend noch teilweise im Banne der Anschauungsweise des Jugendstils. Hammershøis Schlichtheit" und "einfache Konturen" dagegen werden gerade mit dem Komplizierten kontrastiert, wodurch sich unter dem Einfluss Hammershøis anscheinend ein weiteres Abrücken Rilkes vom Jugendstil bemerkbar macht.

Diese Einfachheit und Schlichtheit lassen sich mit besonderer Deutlichkeit in dem Bild beobachten, das in der Ausstellung in Düsseldorf zuallererst Rilkes Interesse an Hammershøi geweckt hatte, nämlich das Porträt Ida Ilsteds. Schlicht und einfach sind an diesem Porträt zunächst die Komposition, die Befestigung der Figur am Rahmen oben und unten, die deutliche, ein bisschen nach links verschobene vertikale Mittelachse und die parallel zur Bildfläche verlaufenden Linien, die den Eindruck des Raumes auf den diffusen Hintergrund reduzieren; dann die Farben, die zwischen dem Schwarz des Kleides und dem Weiß der Halsöffnung eine gleitende Grautonskala durchlaufen, schließlich die ländliche Kleidung der Figur, das schwarze Kleid, die anscheinend etwas zu kleine Joppe und der Hut, der kaum der damaligen Damenmode entsprochen hat, und andeutungsweise der Kleidung einen Anstrich von Kostümierung verleiht.

Der schon hierdurch erweckte Eindruck kindlicher Unschuld und Naivität wird noch dadurch unterstrichen, daß Ida Ilsteds Persönlichkeit eben so schlicht und einfach scheint als der Bildaufbau und ihre Kleidung. Wieder verzichtet Hammershøi auf psychologische Analyse. Der verlorene Blick Idas lässt keineswegs darauf schließen, dass sich in ihrem Innern Gedanken und Gefühle regen, geschweige denn verbalisiert werden. Im Gegenteil, sie scheint in einer eigentümlichen Weise leer, in ihrem leeren Bewusstsein von sich selbst versunken, aber keineswegs ausschließlich nach innen gekehrt, introvertiert. Ihr Blick geht nach außen, aber scheint sich irgendwo im Raume zwischen ihr und dem Betrachter zu verlieren, ohne ihn oder irgendwelchen Gegenstand zu fixieren. Ihr Bewusstsein scheint sich, wie es auch in anderen von Hammershøis Porträts beobachtet werden kann, auf der Kippe zwischen Innen und Außen zu befinden. Abwechselnd scheint sie uns anzuschauen und in 
sich selbst zu versinken, ja manchmal scheinen Außenraum und Innenraum vorübergehend miteinander zu verschmelzen. Hammershøi abstrahiert von seinen persönlichen Gefühlen und von seiner Einsicht in Idas Persönlichkeit und schaut seine Braut einfach als ein Körper mit einem leeren Bewußtsein, das sich kaum von dem die Figur umgebenden Raume unterscheiden läßt. Dadurch ist das Porträt mit Hammershøis Interieurs verwandt, mit den "stillen Stuben", wo Ida Ilsted manchmal als eine Rückwärtsgewandte, identitätslose, von der Leere des Raumes umgebene Figur erscheint. Das Frappante, ja man möchte sagen geradezu Ergreifende dieses Porträts ist es aber, dass es Hammershøi durch Vereinfachung und Abstraktion gelingt, einen Eindruck von Idas rätselhaftem, ihr selbst und dem Beschauer verborgenen Leben zu vermitteln.

Das Porträt Ida Ilsteds unterscheidet sich dadurch markant von den Frauenporträts des Jugendstils. Sie ist nicht durch üppige Ranken und verschnörkelte Ornamente mit ihrer Umgebung verwoben, sondern sitzt in ihrer einfachen Kleidung frei im Raume. Ihr Innenleben - wenn sie ein solches überhaupt besitzt - ist so einfach wie ihr Äußeres. ${ }^{6}$ Obwohl sie keineswegs als geschlechtslos bezeichnet werden kann - der Verlobungsring spricht in dieser Beziehung seine deutliche Sprache - fehlt jede Andeutung des für viele Jugendstilfrauen charakteristischen Kontrastes zwischen Unschuld und erotischer Dämonie. Ida ist keine rätselhafte Arabeske, so wie Musil Jacobsens Marie Grubbe sieht. Ihre Rätselhaftigkeit ist die Rätselhaftigkeit eines reinen unschuldigen Lebens, das Äußeres und Inneres, Raum und Bewusstsein zu einer Einheit verschmilzt. Hier sind wirklich "sinnliche Äquivalente für das Leiseste und Unfaßbarste in uns" gefunden.

Wie das Jacobsenbuch haben sich auch Rilkes Pläne einer Arbeit über Hammershøi vereitelt. Trotzdem ist seine Begegnung mit Hammershøi und seiner Kunst keineswegs eine flüchtige und belanglose Episode geblieben. Im Rahmen von Rilkes Jacobsenrezeption und seiner Rezeption bildender Kunst setzt Hammershøis Kunst wichtige Akzente, die die Bedeutung des Arbeitens, des distanzierten Schauens und der Einfachkeit und Schlichtheit für Rilkes allmähliche Herauslösung aus der Anschauungsweise des Jugendstils unterstreichen. Darüber hinaus hat die wechselseitige Erhellung von Rilkes Gedankenwelt und Hammershøis Kunst eine Herabtönung des Leisen auf ein Innen und Außen, Bewusstsein und Raum umschließendes unfassbares Leben deutlich gemacht, die in aufschlussreicher Weise auf die

\footnotetext{
${ }^{6}$ Ida Ilsted ist keineswegs durch die träumerische Unschuld und ängstliche Sehnsucht nach Leben erfüllt, die die Jugendstilmädchen in Rilkes "Lieder der Mädchen" charakterisieren. Sie erschienen zuerst im Pan, vierter Jahrgang 1898, Viertes Heft, s. 209 - 212. Im Zweiten Heft desselben Jahrgangs, S. 128 - 133, erschien der Aufsatz, "Moderne Kunst in Dänemark. Eine kurze Übersicht" von N.V. Dorph, worin Hammershøi kurz behandelt wird.
} 
Raumperzeption in Rilkes späterer Dichtung vorausdeutet, und zwar auf eine Raumperzeption, die - mit der Cézannes verwandt - sich auch in den von Rilke anscheinend nicht studierten Interieurs Hammershøis geltend macht und die verwandte Modernität des Malers und des Dichters anzudeuten scheint.

\section{Bibliographische Angaben}

RILKE, R. M. Gesammelte Briefe in sechs Bänden. Hrsg. von Ruth Sieber Rilke und Carl Sieber. Leipzig: Insel Verlag $1936-1939$.

VAD, Povl. Vilhelm Hammersh i. Vaerk og Liv. København: Gyldendal, 1988.

WIVEL, Henrik. Vilhelm Hammershøi. København: Søren Fogtdal, 1996.

[Recebido e aceito para publicação em dezembro de 2013]

\section{Rainer Maria Rilke and Vilhelm Hammershøi. On the relationship between visual art and poetry at the turn of the century}

Abstract: The paper examines the impression of two paintings by the Danish painter Vilhelm Hammershøi (1864-1916) on Rilke, "Self-Portrait" from 1889 and "Portrait of a Young Girl" from 1892, which is a portrait of the artist's fiancée Ida Ilstedt. Rilke admired the Danish poet Jens Peter Jacobsen (1847-1885). The kind of looking serves as a common point of reference both of the visual arts and of literature. When he visited Copenhagen during his stay in Sweden in 1904 Rilke was influenced by his admiration for Jens Peter Jacobsen. Rilke had gained access to the art collection of Alfred Bramsen, Hammershøi's patron. Thus he got the opportunity to see others of Hammershøi's works. Here comes the analysis of two paintings. The conscious kind of looking of the two persons was what Rilke was interested in. In "SelfPortrait" a concentrated and objective view; in the Portrait of Ida Ilsted an innocent and meaningless open view somewhere between inside and outside. The two portraits thus thematize the conscious view that Rilke admired at Jacobsen. The works highlight the importance of different kinds of view in later works of Rilke and in the Letters on Cézanne.

Keywords: Rilke. Hammershøi. Jacobsen. Poetry. Visual arts.

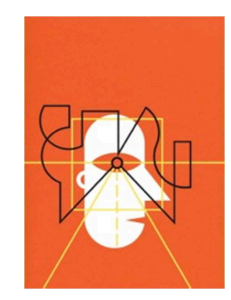

